\title{
Adaptive relaying protocol for wireless energy harvesting and information processing in NOMA systems: Outage probability analysis
}

\author{
Phu Tran Tin ${ }^{1}$, Duy-Hung $\mathrm{Ha}^{2}$, Tran Thanh Trang ${ }^{3}$ \\ ${ }^{1}$ Faculty of Electronics Technology, Industrial University of Ho Chi Minh City, Vietnam \\ ${ }^{2}$ Wireless Communications Research Group, Faculty of Electrical and Electronics Engineering, \\ Ton Duc Thang University, Vietnam \\ ${ }^{3}$ National Key Laboratory of Digital Control and System Engineering, Vietnam
}

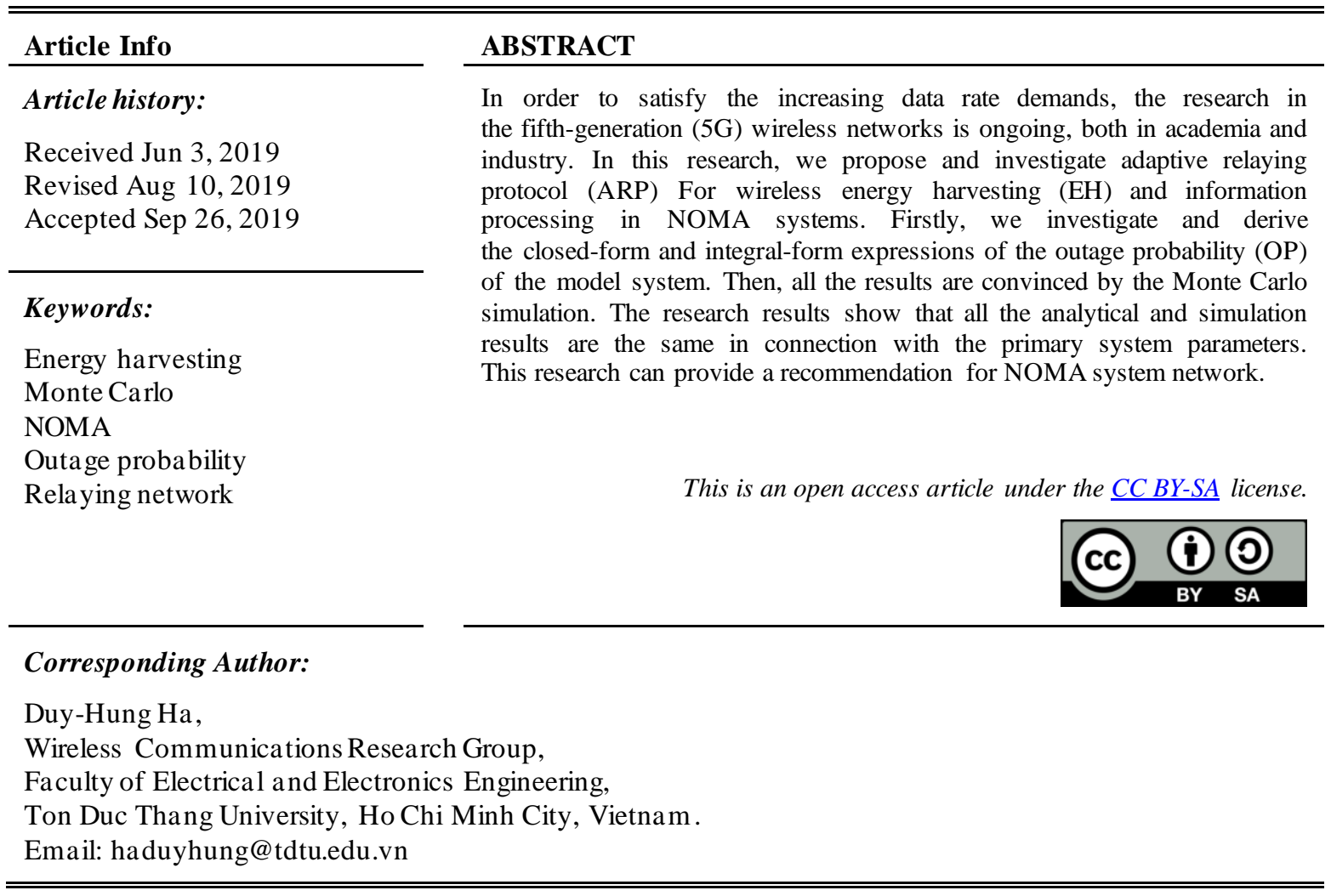

\section{INTRODUCTION}

In order to satisfy the increasing data rate demands, the research in the fifth-generation $(5 \mathrm{G})$ wireless networks is ongoing, both in academia and industry. The full-duplex (FD) is a popular technique in the relaying communication network, which allows the communication node to transmit and receive signals over the same frequency band at the same time slot. In comparison with the traditional water-filling power allocation strategy, NOMA allocates more power to the users with worse channel conditions, which results in a better tradeoff between the system throughput and user fairness [1-6]. From the previous researches, authors in [7] investigate the impact of user pairing on downlink NOMA systems. [8] has proposed power allocation with the max-min fairness criterion. An uplink NOMA scheme with joint power and subcarrier allocations has been considered in [9]. In [10], the authors introduced a cooperation -based NOMA scheme for coordinated direct and relay transmissions. A diversity-oriented detection mechanism for the cooperative relaying system using NOMA has been presented in [11]. The performance of transmit antenna selection for NOMA assisted multiple-input-multiple-output (MIMO) relay networks have been examined in [12]. Authors in [13] presented and investigated a cooperative NOMA transmission scheme. 
In this research, we propose adaptive relaying protocol (ARP) for wireless energy harvesting and information processing in NOMA Systems. Firstly, we investigate and derive the closed-form and integral form expression of the outage probability (OP) of the model system. Then, all the results are convinced by Monte Carlo simulation in connection with all primary system parameters. From the results, we can convince that all the analytical and simulation results are the same in connection with the primary system parameters. The research focuses on some contributions as;

- The ARP for wireless energy harvesting and information processing in NOMA systems is proposed.

- The closed-form and integral-form expressions of the system OP is investigated and derived.

- All the results are convinced by Monte Carlo simulation in connection with all primary system parameters.

\section{SYSTEM MODEL}

The ARP for wireless energy harvesting and information processing in NOMA systems is drawn in Figure 1. In this system model, we denote Source is $S, D_{1}$ and $D_{2}$ are two destination nodes. The energy harvesting (EH) and information processing (IT) for this proposed model system are illustrated in Figure 2. In this protocol, the transmission time $T$ consists of three-time slots. In the first time slot $\alpha \mathrm{T}$ ( $\alpha$ is the time switching factor, $0<\rho<1)$, the energy of the $\mathrm{D}_{1}$ harvest from the source node $\mathrm{S}$. In the second interval time $(1-\alpha) \mathrm{T} / 2$, the source $\mathrm{S}$ transfers the energy and information to $\mathrm{D}_{1}$ at the same time. Finally, the remaining time slot $(1-\alpha) \mathrm{T} / 2$ is used for information transferring from the destination node $\mathrm{D}_{1}$ to the destination node $\mathrm{D}_{2}$.

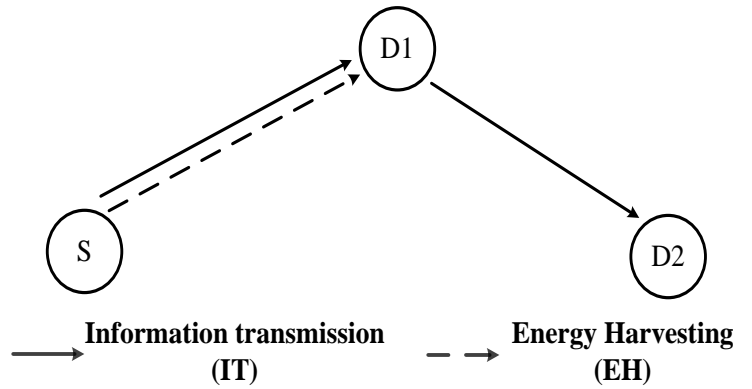

Figure 1. System model

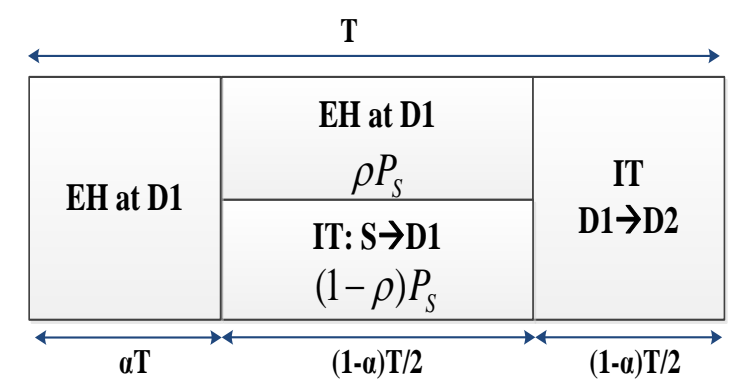

Figure 2. The EH and IT

\subsection{Energy harvesting phase}

The received signal at the relay $\mathrm{R}$ in the first time slot $\alpha \mathrm{T}$ can be formulated as;

$$
y_{D_{1}}^{1}=h\left(\sqrt{b_{1} P_{s}} x_{1}+\sqrt{b_{2} P_{s}} x_{2}\right)+n_{D_{1}}
$$

Where $\mathrm{Ps}_{\mathrm{s}}$ is the transmitting power of source $\mathrm{S}, \mathrm{h}$ is channel gain of $\mathrm{S}_{-} \mathrm{D}_{1}$ link and $\mathrm{n}_{\mathrm{D} 1}$ denoted the additive white Gaussian noise (AWGN) at $\mathrm{D}_{1}$ which has zero-mean and variance $\mathrm{N}_{0}$. $\mathrm{b}_{1}$ and $\mathrm{b}_{2}$ are power allocation coefficients for data symbols $\mathrm{x}_{1}$ and $\mathrm{x}_{2}$ that is wished to send from;

$$
\mathrm{S} \text { toD } \mathrm{D}_{1} \text { and } \mathrm{D}_{2} \text {, respectively and satisfied }\left\{\begin{array}{l}
b_{2}>b_{1}>0 \\
b_{1}+b_{2}=1
\end{array}\right. \text {. }
$$

We assume that $\mathrm{E}\left\{\left|x_{1}\right|^{2}\right\}=\mathrm{E}\left\{\left|x_{2}\right|^{2}\right\}=1$ which $\mathrm{E}\{\bullet\}$ is the expectation operator. Based on (1), the harvested energy in the first time slot $\alpha \mathrm{T}$ can be calculated as;

$$
E_{1}=\eta \alpha T P_{s}|h|^{2}\left(b_{2}+b_{1}\right)=\eta \alpha T P_{s}|h|^{2}
$$

The received signal at $\mathrm{D}_{1}$ in the second interval time $(1-\alpha) \mathrm{T} / 2$ can be expressed as;

$$
y_{D_{1}}^{2}=\sqrt{\rho} h\left(\sqrt{b_{1} P_{s}} x_{1}+\sqrt{b_{1} P_{s}} x_{2}\right)+n_{D_{1}}
$$


Therefore, the harvested energy in the second interval time $(1-\alpha) \mathrm{T} / 2$ can be given by;

$$
E_{2}=\eta \rho\left(\frac{1-\alpha}{2}\right) T P_{s}|h|^{2}\left(b_{1}+b_{2}\right)=\eta \rho\left(\frac{1-\alpha}{2}\right) T P_{s}|h|^{2}
$$

From (2) and (4), the total average transmitted power at the relay node $\mathrm{R}$ can be calculated as the following;

$$
\begin{aligned}
& P_{D_{1}}=\frac{E_{D_{1}}}{(1-\alpha) T / 2}=\frac{E_{1}+E_{2}}{(1-\alpha) T / 2}=\kappa \eta P_{s}|h|^{2} \\
& \text { Where } \kappa=\frac{2 \alpha+(1-\alpha) \rho}{1-\alpha}
\end{aligned}
$$

\subsection{Information transmission phase}

In the second interval time $(1-\alpha) \mathrm{T} / 2$, the received signal at the destination node $\mathrm{D}_{1}$ can be expressed as;

$$
y_{D_{1}}=\sqrt{(1-\rho) P_{s}} h\left(\sqrt{b_{1}} x_{1}+\sqrt{b_{2}} x_{1}\right)+n_{D_{1}}
$$

After receiving the signal from $S, D_{1}$ will decode the signal and decode its own signal by using successive interference cancellation (SIC) [14]. From (6) the received signal to interference plus noise ratio (SINR) at the destination node $\mathrm{D}_{1}$ to detect of the destination node $\mathrm{D}_{2}$ is given by;

$$
\operatorname{SINR}_{2, D_{1}}=\frac{(1-\rho)|h|^{2} b_{2} \Psi}{(1-\rho) \Psi|h|^{2} b_{1}+1}
$$

Where $\Psi=\frac{P_{s}}{N_{0}}$ represents the transmit signal-to-noise ratio (SNR).

After applying SIC, there is no interference remaining in the received signal at the destination node $\mathrm{D}_{1}$. Therefore, the received SNR at the destination node $\mathrm{D}_{1}$ to detect its own signal $x_{1}$ is obtained by;

$$
S N R_{1, D_{1}}=(1-\rho)|h|^{2} b_{1} \Psi
$$

In the third interval time $(1-\alpha) \mathrm{T} / 2$, the decoded signal $x_{2}$ at $\mathrm{D}_{1}$ is forwarded to $\mathrm{D}_{2}$. Hence, the received signal at the destination node $\mathrm{D}_{2}$ can be given as;

$$
y_{D_{2}}=\sqrt{P_{D_{1}}} g x_{2}+n_{D_{2}}
$$

Where $\mathrm{g}$ is channel gain of $\mathrm{D}_{1}-\mathrm{D}_{2}$ link and $n_{D_{2}}$ is AWGN at $\mathrm{D}_{2}$ which has zero-mean and variance $\mathrm{N}_{0}$. Hence, the received $\mathrm{SNR}$ at $\mathrm{D}_{2}$ is given by;

$$
S N R_{2, D_{2}}=\frac{P_{D_{1}}|g|^{2}}{N_{0}}
$$

Substituting (5) into (10), we have:

$$
S N R_{2, D_{2}}=\frac{\kappa \eta P_{s}|h|^{2}|g|^{2}}{N_{0}}=\kappa \eta \Psi|h|^{2}|g|^{2}
$$




\section{SYSTEM PERFORMANCE ANALYSIS}

\subsection{Outage probability (OP) at $D_{1}$}

In the NOMA protocol, $\mathrm{D}_{1}$ is not in an outage when it can decode both $x_{1}$ and $x_{2}$ received from $\mathrm{S}$.

So, the OP at $\mathrm{D}_{1}$ can be expressed as;

$$
O P_{D_{1}}=1-\operatorname{Pr}\left(\operatorname{SINR}_{2, D_{1}}>\gamma_{t h}^{2}, S N R_{1, D_{1}}>\gamma_{t h}^{1}\right)
$$

Where $\gamma_{t h}^{1}=2^{2 R_{1}}-1$ and $\gamma_{t h}^{2}=2^{2 R_{2}}-1$ in which $\mathrm{R}_{1}$ and $\mathrm{R}_{2}$ are the source rates to detect $x_{1}$ and $x_{2}$ at $\mathrm{D}_{1}$, respectively. Substituting (7) and (8) into (12), the $\mathrm{OP}$ at $\mathrm{D}_{1}$ can be rewritten as;

$$
\begin{aligned}
O P_{D_{1}} & =1-\operatorname{Pr}\left(\frac{(1-\rho)|h|^{2} b_{2} \Psi}{(1-\rho) \Psi|h|^{2} b_{1}+1}>\gamma_{t h}^{2},(1-\rho)|h|^{2} b_{1} \Psi>\gamma_{t h}^{1}\right) \\
& =1-\operatorname{Pr}\left[|h|^{2}>\frac{\gamma_{t h}^{2}}{(1-\rho) \Psi\left(b_{2}-\gamma_{t h}^{2} b_{1}\right)},|h|^{2}>\frac{\gamma_{t h}^{1}}{(1-\rho) b_{1} \Psi}\right] \\
& =1-\operatorname{Pr}\left[|h|^{2}>\Theta\right] \\
& =1-\int_{\Theta}^{\infty} f_{|h|^{2}}(x) d x=1-\exp \left(-\lambda_{h} \Theta\right)
\end{aligned}
$$

Where $\Theta=\max \left[\frac{\gamma_{t h}^{2}}{(1-\rho) \Psi\left(b_{2}-\gamma_{t h}^{2} b_{1}\right)}, \frac{\gamma_{t h}^{1}}{(1-\rho) b_{1} \Psi}\right] \quad$ and $\quad \lambda_{h} \quad$ is $\quad$ mean $\quad$ of the random variable $(\mathrm{RV})|h|^{2}$

\subsection{Outage probability (OP) at $\mathrm{D}_{2}$}

$D_{2}$ is in an outage when either $D_{1}$ can not detect $x_{2}$ or $D_{2}$ can not recover the forwarded signal from $\mathrm{D}_{1}$. Hence, the OP at $\mathrm{D}_{2}$ can be obtained as;

$$
O P_{D_{2}}=\operatorname{Pr}\left(\operatorname{SINR}_{2, D_{1}}<\gamma_{t h}^{2}\right)+\operatorname{Pr}\left(\operatorname{SNR}_{2, D_{2}}<\gamma_{t h}^{2}, \operatorname{SINR}_{2, D_{1}}>\gamma_{\text {th }}^{2}\right)
$$

The first term of (14) can be derived as;

$$
\begin{aligned}
\operatorname{Pr}\left(\operatorname{SINR}_{2, D_{1}}<\gamma_{t h}^{2}\right) & =\operatorname{Pr}\left[\frac{(1-\rho)|h|^{2} b_{2} \Psi}{(1-\rho) \Psi|h|^{2} b_{1}+1}<\gamma_{t h}^{2}\right] \\
& =\left\{\begin{array}{l}
\operatorname{Pr}\left[|h|^{2}<\frac{\gamma_{t h}^{2}}{(1-\rho) \Psi\left(b_{2}-\gamma_{t h}^{2} b_{1}\right)}\right], b_{2}-\gamma_{t h}^{2} b_{1}>0 \\
1 \quad, b_{2}-\gamma_{t h}^{2} b_{1} \leq 0
\end{array}\right. \\
& =\left\{\begin{array}{l}
1-\exp \left[-\frac{\gamma_{t h}^{2} \lambda_{h}}{(1-\rho) \Psi\left(b_{2}-\gamma_{t h}^{2} b_{1}\right)}\right], b_{2}-\gamma_{t h}^{2} b_{1}>0 \\
1 \quad, b_{2}-\gamma_{t h}^{2} b_{1} \leq 0
\end{array}\right.
\end{aligned}
$$


The second term of (14) can be rewritten as;

$$
\begin{aligned}
\operatorname{Pr}\left(S N R_{2, D_{2}}<\gamma_{t h}^{2}, S_{\left.N R_{2, D_{1}}>\gamma_{t h}^{2}\right)}\right. & =\operatorname{Pr}\left[\kappa \eta \Psi|h|^{2}|g|^{2}<\gamma_{t h}^{2}, \frac{(1-\rho)|h|^{2} b_{2} \Psi}{(1-\rho) \Psi|h|^{2} b_{1}+1}>\gamma_{t h}^{2}\right] \\
& =\operatorname{Pr}\left[|g|^{2}<\frac{\gamma_{t h}^{2}}{\kappa \eta \Psi|h|^{2}},|h|^{2}>\frac{\gamma_{t h}^{2}}{(1-\rho) \Psi\left(b_{2}-\gamma_{t h}^{2} b_{1}\right)}\right] \\
& =\frac{\gamma_{\gamma_{t h}^{2}}^{2}}{\left.\kappa \eta \Psi h\right|^{2}} \int_{0}^{\infty} f_{|h|^{2}}(x) f_{|g|^{2}}(y) d x d y \\
& =\lambda_{h} \int_{\Xi}^{\infty}\left[1-\exp \left(-\frac{\gamma_{t h}^{2} \lambda_{g}}{\kappa \eta \Psi x}\right)\right] \exp \left(-x \lambda_{h}\right) d x
\end{aligned}
$$

Where $\Xi=\frac{\gamma_{t h}^{2}}{(1-\rho) \Psi\left(b_{2}-\gamma_{t h}^{2} b_{1}\right)}$. Substituting (15), (16) into (14), finally, the OP at $\mathrm{D}_{2}$ can be claimed as;

$$
O P_{D_{2}}=1-\exp \left(-\lambda_{h} \Xi\right)+\lambda_{h} \int_{\Xi}^{\infty}\left[1-\exp \left(-\frac{\gamma_{t h}^{2} \lambda_{g}}{\kappa \eta \Psi x}\right)\right] \exp \left(-x \lambda_{h}\right) d x
$$

\section{NUMERICAL RESULTS AND DISCUSSION}

In this section, we investigate the OP of the model system using Monte Carlo Simulation in connection with the primary system parameters [15-26]. In Figure 3, the effect of $b_{2}$ on the system OP is plotted with the primary system parameters as $\eta=0.8, \mathrm{R}_{1}=\mathrm{R}_{2}=0.5 \mathrm{bps} / \mathrm{Hz}, \alpha=\rho=0.5$. From the results, we can see that the system OP of the destination node $\mathrm{D}_{1}$ significantly decreases with the rising of $b_{2}$. However, the system OP of the destination node $\mathrm{D}_{2}$ decreases when $\mathrm{a}_{2}$ increases from 0.55 to 0.7 and after that, has a massive increase with the remaining values of $b_{2}$. In the same way, the system OP versus $\alpha$ is drawn in Figure 4. In this simulation, we set $\eta=0.8, \psi=7 \mathrm{~dB}, \rho=0.5$, and $b_{2}=0.9, b_{1}=0.1$. As shown in Figure 4, we can state that the OP of the model system has a slight decrease when $\alpha$ varies from 0 to 1 . In both Figure 3 and Figure 4, the simulation and a nalytical results a re the same wit hall values of $\alpha$ and $b_{2}$.

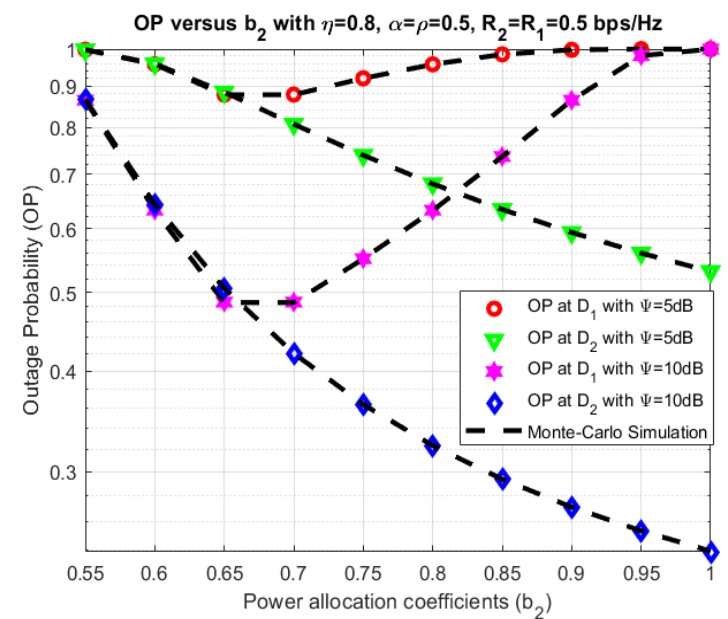

Figure 3. OP versus $b_{2}$

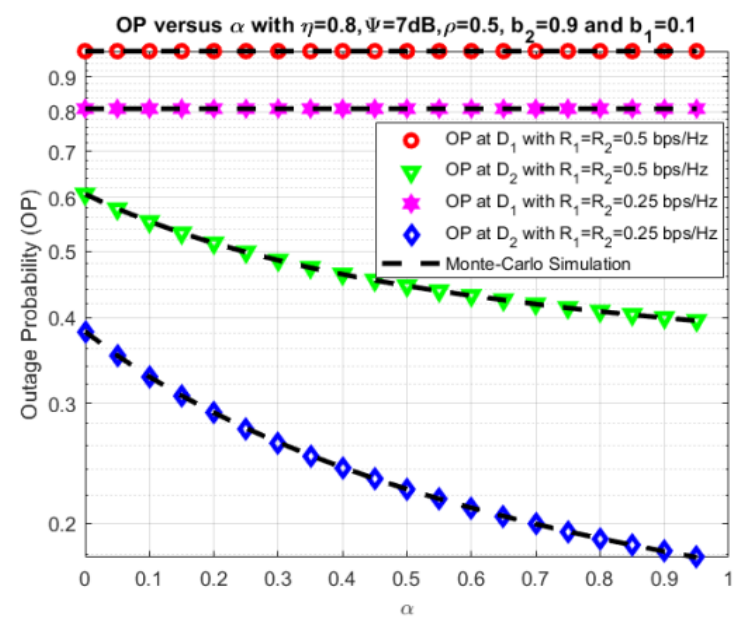

Figure 4. OP versus $\alpha$ 
Furthermore, we investigate the effect of $\eta$ and $\psi$ on the OP of the model system, as plotted in Figure 5 and Figure 6. In these Figure, we set the primary parameters as a $=0.7,0.9, R_{1}=R_{2}=0.5 \mathrm{bps} / \mathrm{Hz}$, $\mathrm{P}_{\mathrm{S}} / \mathrm{N}_{0}=0.5$. From Figure 5 and Figure 6 , it can be observed that the system OP has a slight decrease when $\eta$ varies from 0 to 1 , and the system OP crucially decreases with the rising of $\psi$ from 0 to 25 . In all two Figure, the analytical and simulation results agree well with each other. On another hand, the comparison of the system OP of two destination nodes is demonstrated in all Figure. From the results, we can state that the system $\mathrm{OP}$ of the destination node $\mathrm{D}_{2}$ is better than the destination node $\mathrm{D}_{1}$.

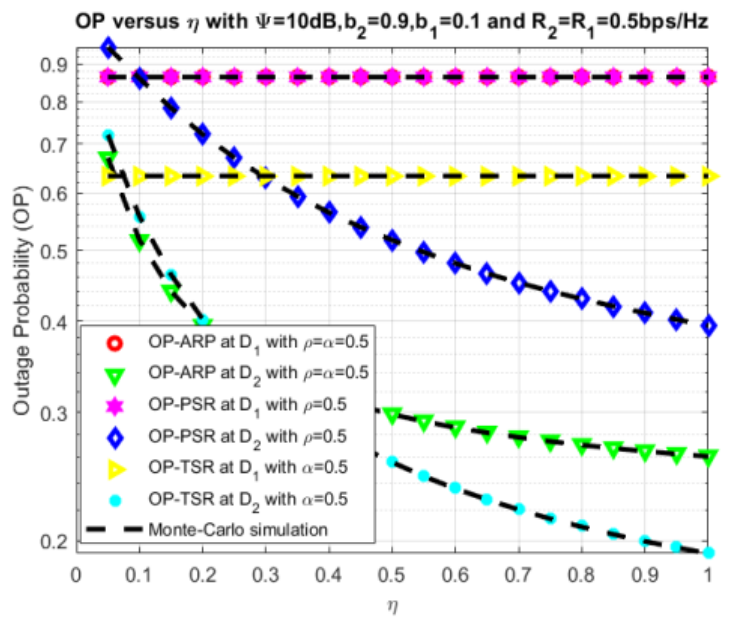

Figure 5. OP versus $\eta$

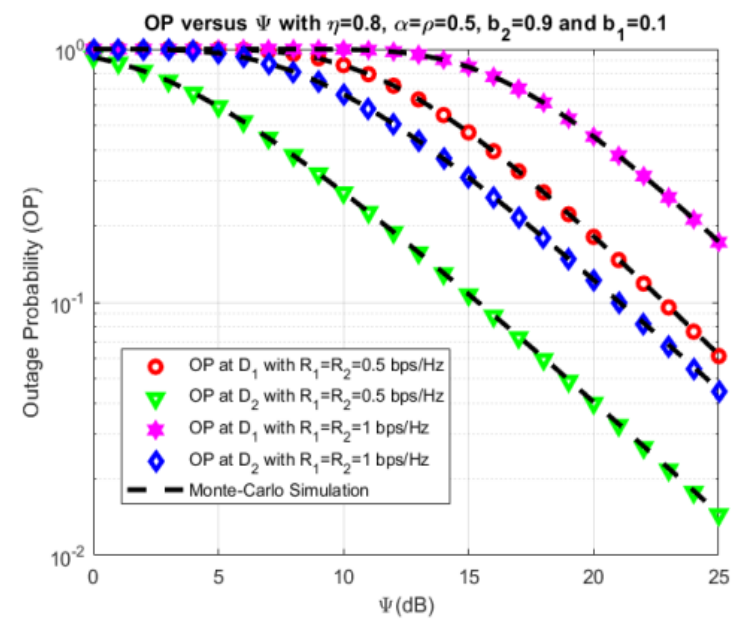

Figure 6. OP versus $\psi$

\section{CONCLUSION}

In this research, we propose the ARP For wireless energy harvesting and information processing in NOMA systems. Firstly, we investigate and derive the closed-form and integral form expression of the outage probability (OP) of the model system. Then, all the results are convinced by Monte Carlo simulation in connection with all primary system parameters. From the results, we can convince that all the analyticaland simulation results are the same in connection with the primary system parameters.

\section{ACKNOWLEDGEMENTS}

This research was supported by National Key Laboratory of Digital Control and System Engineering (DCSELAB), HCMUT, VNU-HCM, Vietnam.

\section{REFERENCES}

[1] S. Bi, C. K. Ho, and R. Zhang, "Wireless powered communication: opportunities and challenges," in IEEE Communications Magazine, vol. 53, no. 4, pp. 117-125, April 2015.

[2] D. Niyato, D. I. Kim, M. Maso, and Z. Han, "Wireless powered communication networks: research directions and technological approaches," in IEEE Wireless Communications, vol. 24, no. 6, pp. 88-97, Dec 2017.

[3] H. Yu, H. Lee, and H. Jeon, "What is 5G? Emerging 5G mobile services and network requirements," Sustainability, vol. 9, no. 1848, pp. 1-22, 2017.

[4] M. Duarte, C. Dick, and A. Sabharwal, "Experiment-driven characterization of full-duplex wireless systems," in IEEE Transactions on Wireless Communications, vol. 11, no. 12, pp. 4296-4307, Dec 2012.

[5] W. C. Y. Lee, "The most spectrum-efficient duplexing system: CDD," in IEEE Communications Magazine, vol. 40, no. 3, pp. 163-166, March 2002.

[6] C. Wang et al., "Cellular architecture and key technologies for 5G wireless communication networks," in IEEE Communications Magazine, vol. 52, no. 2, pp. 122-130, Feb 2014.

[7] Z. Ding, P. Fan, and H. V. Poor, "Impact of user pairing on 5G nonorthogonal multiple-access downlink transmissions," in IEEE Transactions on Vehicular Technology, vol. 65, no. 8, pp. 6010-6023, Aug 2016.

[8] S. Timotheou and I. Krikidis, "Fairness for non-orthogonal multiple access in 5G systems," in IEEE Signal Processing Letters, vol. 22, no. 10, pp. 1647-1651, Oct 2015. 
[9] M. Al-Imari, P. Xiao, M. A. Imran, and R. Tafazolli, "Uplink non-orthogonal multiple access for 5G wireless networks," 11th International Symposium on Wireless Communications Systems (ISWCS), Barcelona, pp. 781-785, 2014.

[10] J. Kim and I. Lee, "Non-orthogonal multiple access in coordinated direct and relay transmission," in IEEE Communications Letters, vol. 19, no. 11, pp. 2037-2040, Nov 2015.

[11] M. Xu, F. Ji, M. Wen, and W. Duan, "Novel receiver design for the cooperative relaying system with non-orthogonal multiple access," in IEEE Communications Letters, vol. 20, no. 8, pp. 1679-1682, Aug 2016.

[12] R. C. Kizilirmak, "Non-orthogonal multiple access (NOMA) for 5G networks," Towards 5G Wireless Networks-A Physical Layer Perspective, pp. 83-98, 2016.

[13] Y. Liu, Z. Ding, M. Elkashlan, and H. V. Poor, "Cooperative non-orthogonal multiple access with simultaneous wireless information and power transfer," in IEEE Journal on Selected Areas in Communications, vol. 34, no. 4, pp. 938-953, April 2016.

[14] S. Luo, R. Zhang, and T. J. Lim, "Optimal save-then-transmit protocol for energy harvesting wireless transmitters," in IEEE Transactions on Wireless Communications, vol. 12, no. 3, pp. 1196-1207, March 2013.

[15] Tan N. Nguyen, T. H. Q. Minh, Phuong T. Tran, and Miroslav Voznak, "Energy harvesting over rician fading channel: A performance analysis for half-duplex bidirectional sensor networks under hardware impairments," Sensors (Basel), vol. 18, no. 6, pp. 1-22, 2018.

[16] Tan N. Nguyen, T. H. Q. Minh, Phuong T. Tran, and Miroslav Voznak, "adaptive energy harvesting relaying protocol for two-way half duplex system network over rician fading channel," Wireless Communications and Mobile Computing, pp. 1-10, 2018.

[17] H. A. Suraweera, G. K. Karagiannidis, and P. J. Smith, "Performance analysis of the dual-hop asymmetric fading channel," in IEEE Transactions on Wireless Communications, vol. 8, no. 6, pp. 2783-2788, June 2009.

[18] I. S. Gradshtein, I. M. Ryzhik, D. Zwillinger, V. H. Moll, and S. Technica Inc., "Table of Integrals, Series, and Products," Academic Press, Waltham, MA, 2015.

[19] M. R. Bhatnagar, "On the capacity of decode-and-forward relaying over rician fading channels," in IEEE Communications Letters, vol. 17, no. 6, pp. 1100-1103, June 2013.

[20] Tan N. Nguyen, T. H. Q. Minh, Phuong T. Tran, Miroslav Voznak, T. T. Duy, Thanh-Long Nguyen, and Phu Tran Tin, "Performance enhancement for energy harvesting based two-way relay protocols in wireless ad-hoc networks with partial and full relay selection methods," Ad hoc Networks, vol. 84, pp. 178-187, Mar 2019.

[21] M. Yazdani, and A. A. Rassafi, "Evaluation of drivers' affectability and satisfaction with black spots warning application," Civil Engineering Journal, vol. 5, no. 3, pp. 576-586, March 2019.

[22] A. A. Astaneh and S. Gheisari, "Review and comparison of routing metrics in cognitive radio networks," Emerging Science Journal, vol. 2, no. 4, pp. 191-201, Aug 2018.

[23] D. Kalistratov, "Wireless video monitoring of the megacities transport infrastructure," Civil Engineering Journal, vol. 5, no. 5, pp. 1033-1040, May 2019.

[24] A. Omidi, R. Karami, P. S. Emadi, and H. Moradi, "Design of the low noise amplifier circuit in band L for improve the gain and circuit stability," Emerging Science Joournal, vol. 1, no. 4, pp. 192-200, Dec 2017.

[25] Phu Tran Trin, Le Anh Vu, Tan N. Nguyen, and Thanh-Long Nguyen, "User selection protocol in DF cooperative networks with hybrid TSR-PSR protocol based full-duplex energy harvesting over rayleigh fading channel: system performance analysis," Indonesian Journal of Electrical Engineering and Computer Science, vol. 13, no. 2, pp. 534-542, 2019.

[26] Tin, Phu Tran, Minh Tran, Tan N. Nguyen, and Thanh-Long Nguyen, "System performance analysis of hybrid time-power switching protocol of EH bidirectional relaying network in amplify-and-forward mode," Indonesian Journal of Electrical Engineering and Computer Science, vol. 14, no. 1, pp. 118-126, 2019. 